\title{
A high-power ultrasonic pulser-receiver for concrete non- destructive testing
}

\author{
Qingbang Han ${ }^{1, a^{*}}$, Youpeng $\mathrm{Xie}^{\mathrm{b}}$, Cheng $\mathrm{Yin}^{\mathrm{c}}$ and Xueping Jiang ${ }^{\mathrm{d}}$ \\ ${ }^{1}$ College of IOT Engineering Changzhou, Jiangsu, 213022,China \\ ahqb0092@163.com, bx_youpeng@qq.com, d84081181@qq.com, angerxing@163.com
}

Keywords: signal acquisition; ultrasonic flaw detection; synchronous signal acquisition.

Abstract. In order to improve the ultrasonic test penetration in the concrete members, a kind of high-power ultrasonic pulser-receiver was designed. With the instantaneous high-voltage pulse by capacitor of charging and discharging, the transformed voltage can reach a peak of $800 \mathrm{v}$. A specific receiver was designed to realize multi-channel synchronous acquisition, storage and data transfer. Experimental results prove that a good SNR (Signal to Noise Ratio) signal can be obtained. Along with other advantages such as easy manufacture and operation, cost efficiency, the proposed scheme may have a wide application in the non-destructive ultrasonic evaluation of concrete members.

\section{Introduction}

One of the main goal of nondestructive testing in civil engineering is a reliable assessment of the integrity of concrete members, where the detection methods based on ultrasonic waves are frequently applied. The implementation of ultrasonic techniques for concrete evaluation has been hampered by the heterogeneous nature of concrete, which leads to the scattering or attenuation of stress waves and limits the penetration of the stress waves generated by a conventional electro-acoustic transmitter [1-3]. The present high voltage impulse generator such as panametrics 5058PR etc are not suitable for field measurement for its disadvantage of valuableness and heaviness. In order to break the bottleneck in the application of ultrasonic techniques, a high-power ultrasonic pulser-receiver based on principle of charge-discharge and voltage transformation was designed in this paper. Fig. 1 plots the schematic diagram of the high-power ultrasonic pulser-receiver systems, which consists of a pulser and a receiver.

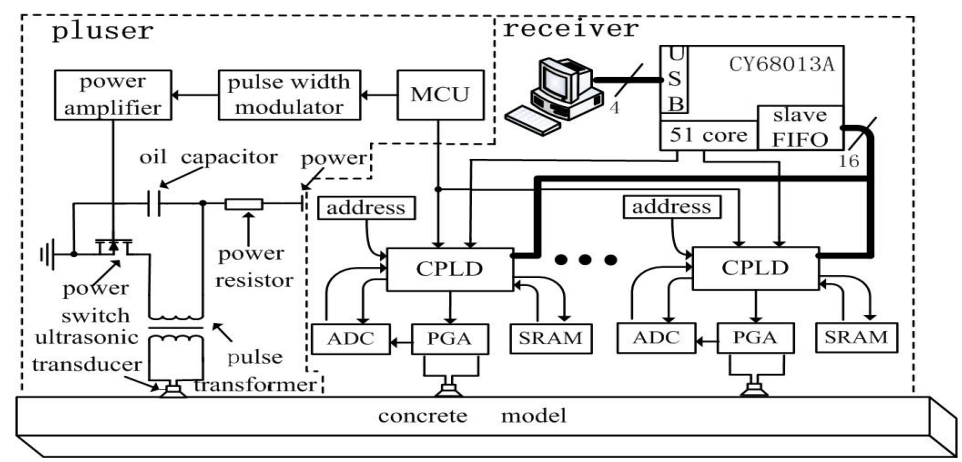

Fig. 1 Schematic diagram of the system

\section{The design of pulser.}

This high-power pulser circuit consists of Micro Control Unit (MCU), the power switch, pulse width modulator, power amplifier, power resistor, oil capacitor, and pulse transformer. Pulse Width Modulation (PWM) generated by MCU is adjusted by the pulse width modulator through the power amplifier, the output signal is strong enough to drive the power switch to control the charge and discharge process of the oil capacitor, and a high-voltage pulse signal to excite ultrasonic transducer can be generated with the help of pulse transformer.

To get a shorter pulse, the time constant $\tau(=\mathrm{RC})$ of the circuit should be small, i.e. the capacitance is smaller. However, the small capacitance will lead to a decreased electric quantity, along with a 
reduced rated voltage range of the capacitor, and finally limit the pulse amplitude. In our design, the capacitor is chosen $20 \mathrm{uF}$, and the value of rated voltage is in range of $0-630 \mathrm{~V}$.

The pulser should change the state of switch quickly to achieve charging and discharging of the capacitor. It is of great importance to choose a suitable power switch that has the ability to quickly switch. Insulated Gate Bipolar Transistor(IGBT) is a kind of power device that consists of Bipolar Junction Transistor(BJT) and MOS transistor. It is selected as the power switch due to its advantage of low driven power, low saturation voltage and fast switching speed[4-5]. In this design, the module of $1 \mathrm{HW} 25 \mathrm{~N} 120 \mathrm{R} 2$ IGBT was applied, which could support the maximum current of $30 \mathrm{~A}$ and the permissible voltage of $300 \mathrm{~V}$.

As the current from MCU is too weak to drive the power switch, a low noise operational amplifier (NE5532) is used to enhance the current.

The design of pulse transformer is vital to the high-power pulser. The power, the nature of magnetic material, geometry and size of the transformer will satisfy the follow expression:

$$
A_{c} \times A_{e}=\frac{P_{t} \times 10^{4}}{42 f B_{m} K_{o} K_{j}} \times 1.16
$$

Where ${ }^{B_{m}}$ is the flux density; ${ }_{c}$ is the effective cross-sectional area of the core; $A_{e}$ is the window area of the core; $P_{t}$ is the power of the transformer, $f$ is the operating frequency; $K_{o}$ is the window duty factor; where the $K_{j}$ is the current density factor of the winding.

The numbers of turns are determined by

$$
N_{1}=\frac{V_{1}}{4 f B_{m} A_{e}}
$$

Where V1 represents input voltage. The pulse transformer turns ratio is set as 1:10 in this design.

\section{The design of receiver.}

To meet the needs of signal process, the receiver which enable multi-channel synchronous collect consists of CPLD(Complex Programmable Logic Device), PGA(Programmable Gain Amplifer), address, SRAM(Static Random Access Memory), and USB communication modules. The CPLD was chosen as the core chip for its cost efficiency and easy to coordinate AD converter、SRAM and FIFO(First In First Out); The signal amplifier ensures the minimum of the received signal is limited in the acquisition range of the $\mathrm{AD}$ converter; the SRAM were used to store data temporarily then deliver it to PC.

At the moment of the trigger command was sent by MCU, all the detection signals were collected and stored in SRAM. The IO ports of each CPLD are directly connected with slave FIFO interface of CY7C68013A to provide a data bus for transmitting data to PC. At the initial state, the dates could not be transmitted because each CPLD data ports are in high-impedance state. To avoid channel congestion, when the data transfer instructions are sent by PC, only the data IO ports of one CPLD change their state from high-impedance to conductance while other ports of CPLD still in high-impedance state, the corresponding data stored in SRAM are access to the PC, after the corresponding data stored in SRAM are access to the PC, these IO ports return to be obstructed and other CPLD transmit data in turn. Although the data are transmitted in different time, the data acquisition at start time is accomplished simultaneously, so the synchronizing signals can be obtained from the PC.

CY7C68013A is used to translate control commands and data with PC[6]. To ensure the high-speed data transmission, the 51 core in the CY7C68013A was prohibited to participate in data transmission and only carry out the control command code. 
The PWM generated by MCU is used not only to drive the power switch to generate a single pulse signal, but also to act as signal acquisition synchronization that ensures the validity of the data collection. A synchronizer trigger was designed in CPLD to realize simultaneous acquisition.

\section{Measurement results and discussion.}

Experiments were carried out to test high-power ultrasonic pulser-receiver discussed in this paper is shown in Fig.2. Fig. 2a shows the aforesaid pulser and receiver. We tested the signal produced by the high-power single-pulse directly; a near-perfect single pulse signal collected with oscilloscope (Tektronix DPO3034) was shown in Fig. 2b. By using high frequency current test loop (PINTCH PT-7802 ) and high-frequency voltage probe (PINTCH PT-5150), the test results on the condition of $10 \mathrm{~V}$ external voltage are: the maximum, instantaneous power is $142 \mathrm{~W}$; the maximum instantaneous voltage can reach $800 \mathrm{~V}$; the pulse width is in range of $25 \mathrm{us}$ to $300 \mathrm{us}$. The amplitude of power and instantaneous voltage of the device are obviously higher than that of general ultrasonic pulse signal pulser, for example, the Olympus pulser and receiver 5073, pulse amplitude is about 180v, power is about in the tens of watts magnitude.

To test receiving system, a test sample of a cubic concrete block with $25 \mathrm{~cm} 3$ was applied, and a corrugated pipe is located in block is shown in Fig. 2c. Using the pulse to produce an ultrasonic wave in concrete, a receiving transducer ultrasonic signal translates the stress into an electric signal, which is collected by ultrasonic receiver is showne in Fig. $2 \mathrm{~d}$.

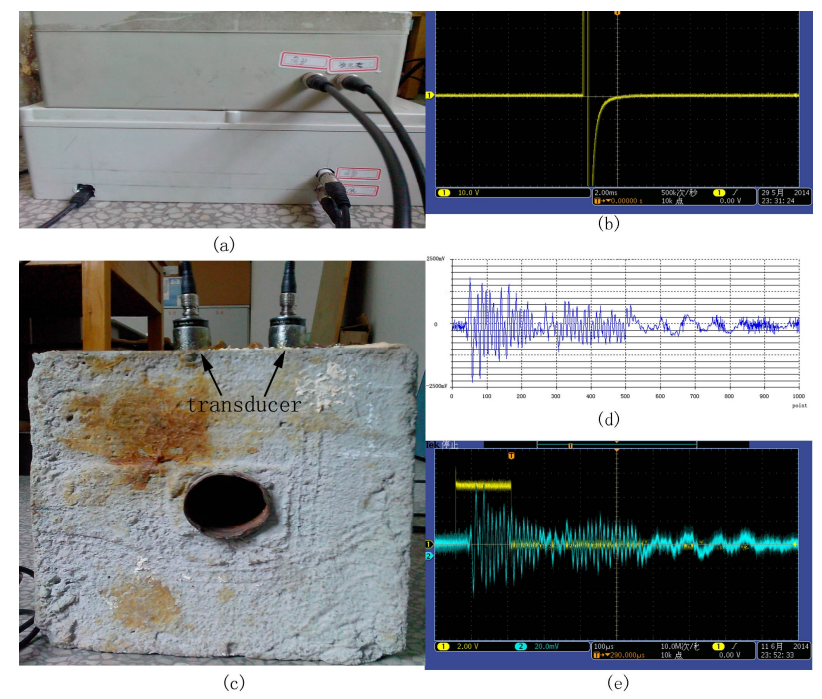

Fig. 2 Experimental arrangement for device test

a The device of the high-power ultrasonic pulser-receiver; $\mathbf{b}$ High-power single-pulse signal; $\mathbf{c}$ The concrete members model for testing; d Ultrasonic signal collected by pulser-receiver ; e.Ultrasonic signal collected by Tektronix oscilloscope DPO3034

Compared with the waveform obtained by the Tektronix DPO3034 oscilloscope showed in Fig. 2e, we find they are almost the same in shape, but a little different in voltage amplitude; however, the receiver proposed in this paper can magnify the received signal with PGA to recognize the weaker signals. In addition, all collected signals are synchronous, which will be conducive to judge the arriving time of each wave.

As an example, an actual measurement was conducted to concrete member as seen in Fig. 3, the purpose is to test corrugated pipe grouting defects. Because the weak signal reflected by grouting defects are usually impossible to discern in the background of the strong scatteringscattering of concrete member, the conventional ultrasonic pulser-receiver can not obtain the desired signals[7]. However, by using our instrument, the good SNR signal could be found is shown in Fig.4, and each 
wave could be identified according their arriving time owing to the synchronize, which will help us to analyze signals and extract characterization of defect.

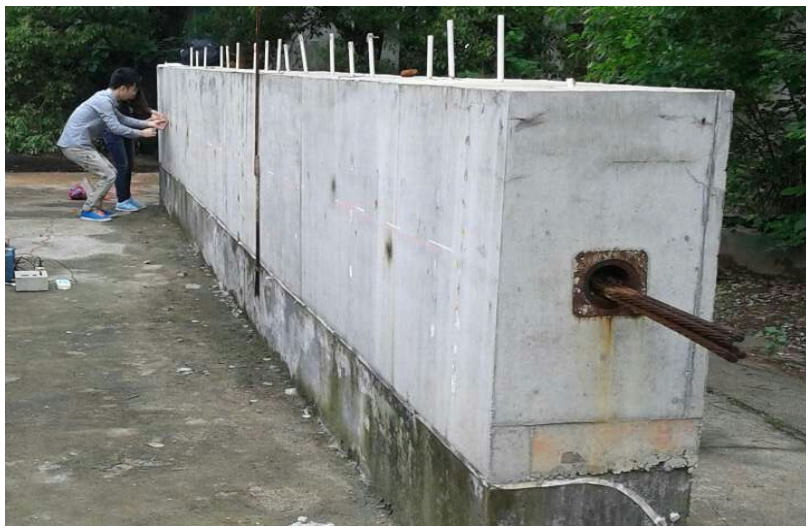

Fig. 3 The actual concrete member with corrugated pipe

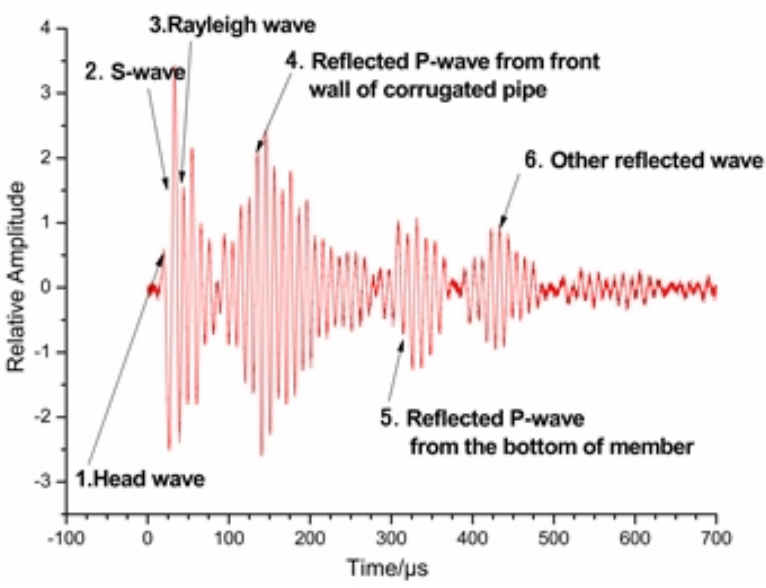

Fig. 4 The transient respond of collected signal

\section{Conclusion.}

In this paper, a high-power ultrasonic detection device is designed. Ultrasonic pulser generates a pulse signal by controlling a capacitor charging and discharging, and the pulse signal produces instantaneous high-voltage pulse after it goes through a transformer. CPLD is applied as the core control chip for the receiver, which consists of the synchronizing and dress, the PGA, the SDRAM and the communication module. The experimental results confirm a better performance when compared with the present apparatus for concrete members ultrasonic testing. Other advantages of the system such as easy to construct, cost less, and be convenient to field operate makes it possible to improve the effectiveness and practicability to ultrasonic testing of concrete members.

\section{Acknowledgement.}

This work were supported by National Natural Science Foundation of China (No.11274091, 61302124)and the Funds for the Central Universities of Hohai university(Grant No. 2011B11014)

\section{References}

[1]Accu-Tron Inc.: 'Ultrasonic pulser-receiver introduced'.NDT International, Volume 21, Issue 3, June 1988, Page 185

[2]N. Ruiz, P. Vera, J. Curpian, D. Martinez and R. Mata.: 'Matching pursuit-based signal processing method to improve ultrasonic flaw detection in NDT applications', ELECTRONICS LETTERS 20th February 2003 Vol. 39 No. 4

[3] Indexing terms: Acoustic-surface-wave devices, Ultrasonic delay lines, Radar equipment.: 'Use of a surface-acoustic-wave delay line to provide pseudocoherence in a clutter reference pulse doppler radar', ELECTRONICS LETTERS 25th January 1973 Vol. 9 No. 2 
[4]V.K. Sundaramoorthy, E. Bianda, R. Bloch, et al.: 'A study on IGBT junction temperature (Tj) online estimation using gate-emitter voltage (Vge) at turn-off Original Research Article Microelectronics Reliability', In Press, Corrected Proof, Available online 26 June 2014

[5]Hojong Choi , K. Kirk Shung .: 'Novel power MOSFET-based expander for high frequency ultrasound systems',Ultrasonics 54 (2014) 121 - 130

[6]Cypress EZ-USB FX2, Technical Reference Manual

[7]Philip E. Bloomfield ::'Extensions of the scattering-object function and the pulser-receiver impulse response in the field II formalism', Ultrasonics, Volume 43, Issue 6, May 2005, Pages 421-427 